\title{
Mesoscale Patterns Formed by Evaporation of a Polymer Solution in the Proximity of a Sphere on a Smooth Substrate: Molecular Weight and Curvature Effects
}

\author{
Suck Won Hong, ${ }^{\dagger}$ Jianfeng Xia, ${ }^{\dagger}$ Myunghwan Byun, ${ }^{\dagger}$ Qingze Zou, ${ }^{\dagger}$ and Zhiqun Lin ${ }^{*} \dagger$ \\ Department of Materials Science and Engineering, Iowa State University, Ames, Iowa 50011, and \\ Department of Mechanical Engineering, Iowa State University, Ames, Iowa 50011
}

Received January 10, 2007; Revised Manuscript Received February 13, 2007

\begin{abstract}
A drop of polymer solution was constrained in a sphere-on-flat geometry, resulting in a liquid capillary bridge. As solvent evaporated, intriguing surface patterns of polymer formed, which were strongly dependent on the molecular weight (MW) of polymer. Dotted arrays were formed at low MW; concentric rings were produced at intermediate MW; concentric rings, rings with fingers, and punch-hole-like structures, however, were yielded at high MW. Rings with fingers as well as punch-hole-like structures were manifestations of simultaneous occurrence of the "stick-slip" motion of the contact line and the fingering instabilities of rings. In addition, the curvature of the sphere in the sphere-on-flat geometry was found to affect the pattern formation. A decrease in the curvature of the sphere led to an earlier onset of the formation of punch-hole-like structures when high-MW polymer was employed as the nonvolatile solute.
\end{abstract}

\section{Introduction}

Dissipative structures, such as convection patterns ${ }^{1-4}$ and fingering instabilities, ${ }^{5-7}$ are formed when a droplet containing nonvolatile solutes (e.g., polymers, nanoparticles, colloids, or DNA) is allowed to evaporate on a solid surface. ${ }^{8,9}$ However, these self-organized structures are, in general, irregular. The evaporation is, in principle, a nonequilibrium process. ${ }^{9}$ Therefore, to fully exploit the dynamic self-assembly via irreversible solvent evaporation as a simple, lithography- and external-fieldfree route to well-ordered mesoscale structures that may have potential technological applications, it requires delicate control over the evaporation process and the associated capillary flow. To this end, several elegant methods have emerged. ${ }^{8,10,11}$ Recently, regular polymer pattern have been produced continuously from a receding meniscus, formed between two parallel plates, by controlling the speed of the upper sliding plate at a constant velocity while keeping the lower plate stationary. ${ }^{8}$ In our previous work, we reported that concentric rings of electrically conducting polymer and organometallic polymer of high regularity were formed naturally and spontaneously via controlled, repetitive "stick-slip" motion of the three-phase contact line when a drop of polymer solution was confined either between two crossed cylindrical mounts covered with single crystals of mica sheets ${ }^{10}$ or between a spherical lens made of silica and a Si substrate (sphere-on-flat geometry), resulting in a capillary-held polymer solution (i.e., capillary bridge). ${ }^{1-17}$ The evaporation in this geometry was restricted to the edge of the droplet, and the "stick-slip" cycles resulted in hundreds of concentric rings with regular spacing, very much resembling a miniature archery target. Each ring was nanometers high and several microns wide. ${ }^{10-12}$ By tuning the interfacial interaction between the polymer and the substrate that governed the stability of thin films, intriguing, ordered dissipative structures can be produced as a result of synergy of controlled self-assemblies

* To whom correspondence should be addressed. E-mail: zqlin@ iastate.edu.

Department of Materials Science and Engineering.

$\doteqdot$ Department of Mechanical Engineering. of the polymer and its destabilization mediated by the interfacial interaction. $^{15}$

We have reported that the use of solutions with different concentrations and different solvents effectively mediated the pattern formation in an evaporating droplet containing nonvolatile solutes. ${ }^{11}$ In this paper, we extend our previous work to investigate the molecular weight (MW) effect on the mesoscale polymer patterns formed by drying a drop of polymer solution in a sphere-on-flat geometry (i.e., a spherical lens (or a pushpin) on a Si substrate), as depicted in Figure 1. The patterns, ranging from dotted arrays at low MW to concentric rings at intermediate $\mathrm{MW}$ to concentric rings and punch-hole-like structures at high MW, were observed. A qualitative explanation was given to understand the pattern formation. Moreover, the curvature effect of the sphere was also studied by replacing the spherical lens (radius of curvature, $R \sim 2.0 \mathrm{~cm}$ ) with the pushpin $(R \sim 2.5 \mathrm{~cm})$. As the curvature decreased (i.e., from $1 / R \sim$ $1 / 2.0 \mathrm{~cm}^{-1}$ to $1 / 2.5 \mathrm{~cm}^{-1}$ ), represented as a decrease in the distance between the sphere and $\mathrm{Si}$, an earlier onset of fingering instabilities of polymer was observed owing to a reduction in the velocity of the displacement of the meniscus (i.e., the liquidvapor interface) in the capillary bridge.

\section{Experimental Section}

Materials. Four polystyrene homopolymers (PS) (Polymer Source, Inc.) with different molecular weight were used in the studies. The number-average MW, $M_{\mathrm{n}}$ (and weight-average MW, $\left.M_{\mathrm{W}}\right)$, of PS were $60 \mathrm{~K}(62.5 \mathrm{~K}), 112 \mathrm{~K}(118 \mathrm{~K}), 420 \mathrm{~K}(483 \mathrm{~K})$, and $876 \mathrm{~K}(1050 \mathrm{~K})$. These four PS are denoted PS-60K, PS-112K, PS$420 \mathrm{~K}$, and PS-876K, respectively. All PS were dissolved in toluene to prepare the PS toluene solutions at the concentration of 0.25 $\mathrm{mg} / \mathrm{mL}$. Subsequently, the solutions were purified with $0.2 \mu \mathrm{m}$ hydrophobic membrane filter.

The spherical lens made of fused silica with a radius of curvature, $R \sim 2.0 \mathrm{~cm}$, the push-pin made from stainless steel with $R \sim 2.5$ $\mathrm{cm}$, and silicon wafers were cleaned by the mixture of sulfuric acid and Nochromix. Subsequently, they were rinsed with DI water extensively and blow-dried with $\mathrm{N}_{2}$.

Sample Preparation. To construct a confined geometry, a spherical lens (or a push-pin) and a Si wafer were used. The sphere 


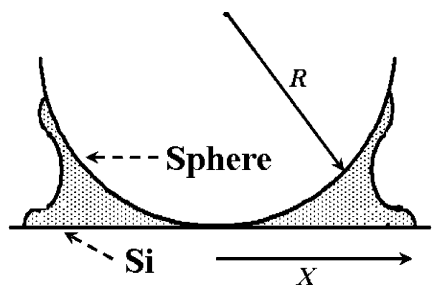

Figure 1. Schematic illustration of a drop of polymer solution placed between sphere and $\mathrm{Si}$ substrate (i.e., sphere-on-flat geometry), forming a capillary-held liquid bridge. The radius of curvature of upper sphere is $R$. The absolute position of the ring away from the sphere/Si contact center is $X$.

(i.e., the spherical lens or the push-pin) and Si were firmly fixed at the top and bottom of sample holders inside a sealed chamber, respectively. To implement a confined geometry, an inchworm motor with a step motion of a few micrometers was used to place the upper sphere into contact with the lower stationary Si substrate. Before they contacted (i.e., separated by approximately a few hundred micrometers apart), a drop of $\sim 23 \mu \mathrm{L}$ PS toluene solutions was loaded and trapped within the gap between the sphere and $\mathrm{Si}$ due to the capillary force. The sphere was finally brought into contact with Si substrate by the inchworm motor such that a capillary-held PS solution formed with evaporation rate highest at the extremity (Figure 1). It is noteworthy that the use of a sealed chamber ensured a stable solvent evaporation against possible external influences such as the air convection and the humidity in an open space.

The evaporation took about half an hour to complete. Afterward, the sphere and $\mathrm{Si}$ were separated. The intriguing structures were produced on both the sphere and Si surfaces. Because of the curving effect of the sphere, only the patterns formed on Si were evaluated by optical microscope (OM; Olympus BX51 in the reflection mode) and atomic force microscopy (AFM; Dimension 3100 scanning force microscope in the tapping mode (Digital Instruments)). BStap300 tips (Budget Sensors) with spring constants ranging from 20 to $75 \mathrm{~N} / \mathrm{m}$ were used as scanning probes.

\section{Results and Discussion}

1. Molecular Weight Effect. The structures shown in Figures 2-4 were obtained by drying the four PS toluene solutions placed between the spherical lens $(R \sim 2 \mathrm{~cm})$ and Si substrate. The evaporation took place under controlled conditions (i.e., constant temperature (room temperature) and the same initial polymer concentration, $c=0.25 \mathrm{mg} / \mathrm{mL}$ ). For the PS with the MW of $60 \mathrm{~K}$, irregular dotted arrays were formed exclusively on Si substrate by drying the PS-60K toluene solution in the sphere-on-Si geometry (Figure 1). A typical optical micrograph of randomly distributed PS-60K aggregates is shown in Figure 2a. The average height of PS-60K aggregates was 204 and 126 $\mathrm{nm}$ for bigger and smaller PS dots, respectively (Figure 2b). It is important to note that similar patterns were observed for other PS-60K samples at different concentrations (from 0.125 to 5 $\mathrm{mg} / \mathrm{mL}$ ), suggesting that (a) the formation of isolated, randomly dispersed PS-60K aggregates was governed by the dewetting ${ }^{8}$ and (b) the force exerted by the deposition of PS-60K was not strong enough to pin the three-phase contact line (i.e., form a "coffee ring"). ${ }^{18-20}$ Thus, the thin liquid film ruptured on the surfaces into randomly distributed PS dots to minimize the surface energy. ${ }^{5,8}$

When a higher MW PS was used (i.e., PS-112K), microscopic concentric rings of PS-112K were obtained as shown in Figure 3a. The formation of concentric rings was a direct consequence of repetitive "stick-slip" motion of the contact line (i.e., the competition between the pinning force ("stick") and the depinning force ("slip")) toward the center of sphere/Si contact
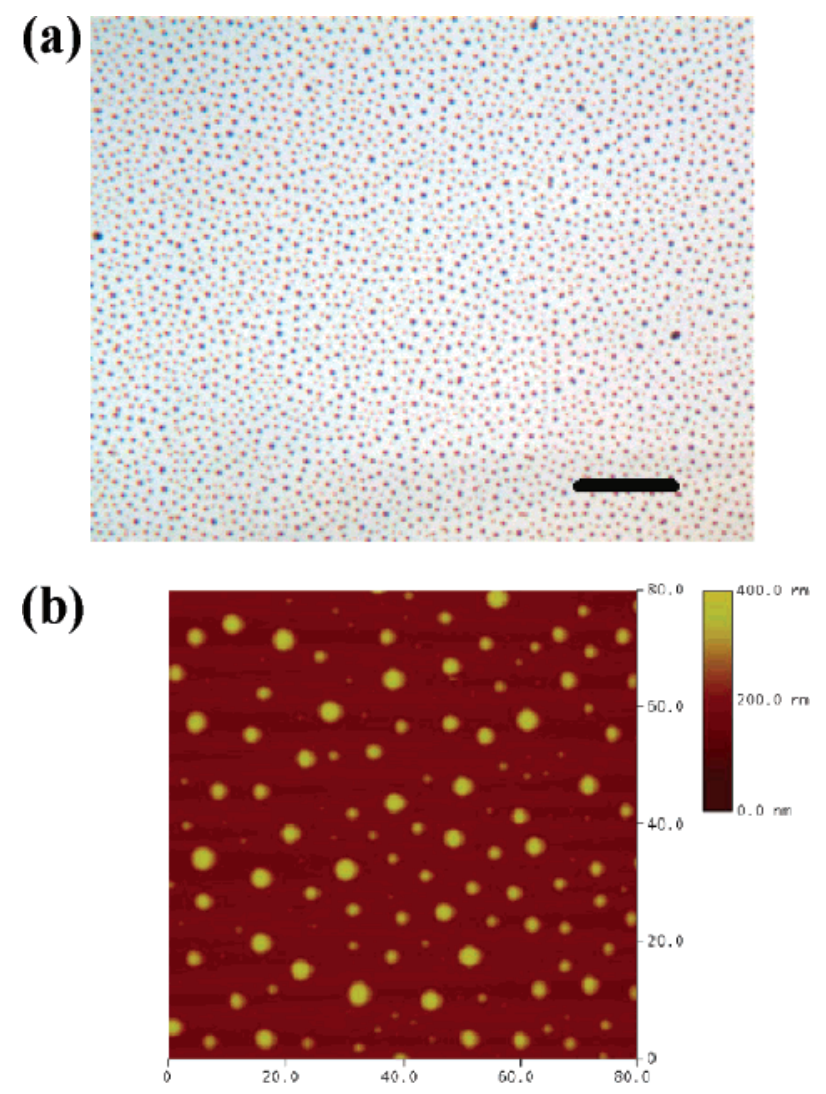

Figure 2. (a) Optical micrograph of randomly dispersed PS-60K aggregates on Si substrate after the evaporation of toluene in the sphereon-flat geometry. The initial concentration of the PS-60K toluene solution, $c$, is $0.25 \mathrm{mg} / \mathrm{mL}$. Scale bar $=25 \mu \mathrm{m}$. (b) AFM image of PS-60K aggregates, showing the details of dewetted patterns. The image size is $80 \mu \mathrm{m} \times 80 \mu \mathrm{m}$.

with elapsed time as discussed in our previous work. ${ }^{10-12,15}$ The solution front was arrested at the capillary edge as toluene evaporated (Figure 1). The local viscosity of the contact line was then increased with time. This led to the vitrification of a PS-112K ring before the solution front jumped to next position where it was arrested again. ${ }^{21}$ The average jumping distance (i.e., the center-to-center distance between adjacent rings), $\lambda_{\mathrm{C}-\mathrm{C}}$, and the average height of the ring, $h$, are $30.2 \mu \mathrm{m}$ and $228 \mathrm{~nm}$, respectively, as determined by AFM (Figure $3 b$ ). Locally, the rings appeared as parallel stripes, and the shape of each ring was, however, nonuniform.

A set of intriguing surface patterns emerged when the PS with the MW of $420 K$ (i.e., PS-420K) was employed. Figure 4a shows an optical micrograph of the pattern of PS-420K produced at different stages of "stick-slip" motion of the contact line as toluene evaporated from the capillary edge. The 2D AFM height images of surface patterns, roughly corresponding to the locations in the upper left, middle, and lower right of the optical micrograph in Figure $4 a$, are shown in Figure $4 b-d$. The concentric rings and rings with fingering instabilities ${ }^{5-7}$ were formed at distances far away from the center of sphere/Si contact (i.e., at larger $X$ ) (upper left of optical micrograph in Figure 4a and $4 \mathrm{~b}$ ). The fingering instabilities are represented as the surface perturbation with a well-defined wavelength at edges of a ring. The fingers appeared on both sides of a ring at intermediate $X$ (Figure 4c). When the solution front was closer to the center of sphere/Si contact (i.e., at small $X$ ), the punch-hole-like structures were produced by interconnecting the fingers from adjacent rings (Figure 4d,e). The characteristic distance between adjacent PS$420 \mathrm{~K}$ fingers on a ring, $\lambda_{\mathrm{F}}$, and the height of the ring, $h$, were 


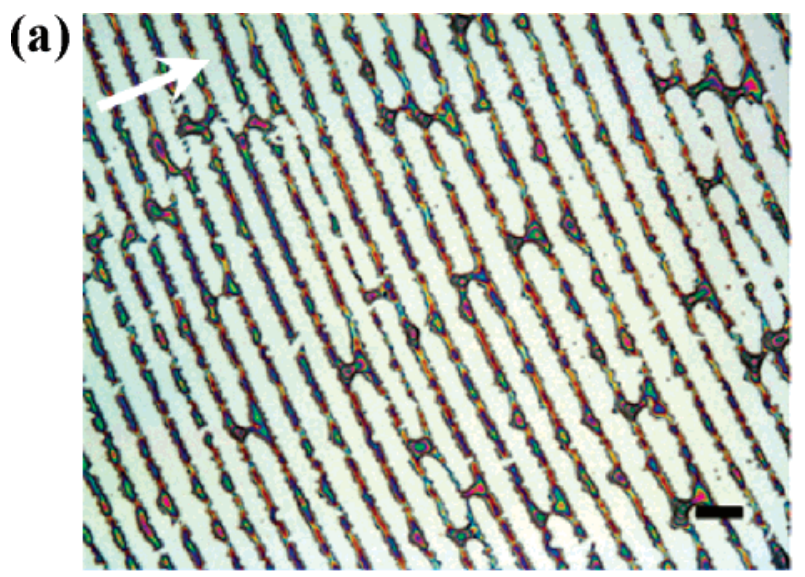

(b)

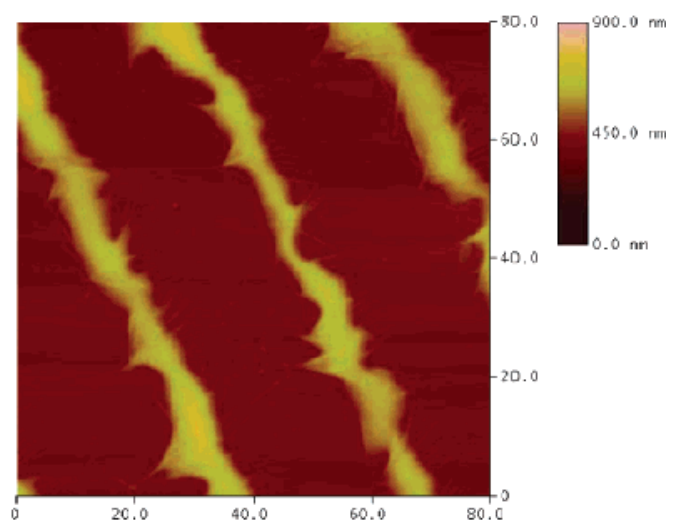

Figure 3. (a) Optical micrograph of concentric ring patterns of PS$112 \mathrm{~K}$ on Si substrate after the evaporation of toluene in the sphereon-flat geometry. The initial concentration of the PS- $112 \mathrm{~K}$ toluene solution, $c$, is $0.25 \mathrm{mg} / \mathrm{mL}$. Scale bar $=50 \mu \mathrm{m}$. The arrow on the upper left marks the direction of the movement of the solution front. (b) A typical 2D AFM height image of PS- $112 \mathrm{~K}$ rings. The image size is 80 $\mu \mathrm{m} \times 80 \mu \mathrm{m}$.

$25.35 \mu \mathrm{m}$ and $379 \mathrm{~nm}$ at $X=3190 \mu \mathrm{m}$ (Figure 4b), $25.26 \mu \mathrm{m}$ and $335 \mathrm{~nm}$ at $X=3090 \mu \mathrm{m}$ (Figure 4c), and $24.90 \mu \mathrm{m}$ and $327 \mathrm{~nm}$ at $X=2950 \mu \mathrm{m}$ (Figure 4d). The average width and height of fingers at the center connecting two adjacent rings were $3.74 \mu \mathrm{m}$ and $195 \mathrm{~nm}$ (Figure 4d,e).

The emergence of PS-420K surface patterns from rings to rings having fingering instabilities to punch-hole-like structures has been qualitatively understood on the basis of the fact that the velocity of the displacement of the meniscus at the capillary edge, $v$, was inversely proportional to the distance from the capillary entrance to the meniscus. ${ }^{15,22}$ A faster $v$ stabilized the front, while a slower $v$ led to the development of fingering instabilities at a propagating front. ${ }^{23}$ As the solution front progressed toward the center of the sphere/Si contact, $v$ decreased owing to a decrease in the evaporation rate of toluene. As a result, fewer PS-420K were available to transport and pin the contact line. This caused the formation of fingering instabilities. The center-to-center distance between two adjacent rings, $\lambda_{\mathrm{C}-\mathrm{C}}$, decreased gradually as the solution front approached the center of sphere/Si contact. This facilitated the fingers from adjacent rings to connect each other. As a result, the sequence of microscopic holes was produced with increasing proximity to the center of sphere/Si contact (low right of the optical micrograph in Figure $4 \mathrm{a}$ and $4 \mathrm{~d}-\mathrm{e}$ ). Similar surface patterns were observed from the drying of $0.25 \mathrm{mg} / \mathrm{mL}$ PS-876K toluene solution, i.e., forming rings, rings with fingers, and a periodic array of punch-holes progressively with a decrease in $X$ (Figure $1)$.
We now turn our attention to further address qualitatively the molecular weight effect on the structure formation based on the overlap concentration argument. de Gennes et al. presented three concentration regimes for polymer random coils in solution; they are dilute, semidilute, and concentrated solutions, corresponding to separated chains, overlapping chains, and entangled chains, respectively. ${ }^{24,25}$ The overlap concentration, $C^{*}$, from dilute to semidilute solution is defined as the concentration at which the polymer coils touch each other. ${ }^{24,26}$

$$
C^{*}=\frac{3 M}{4 \pi R_{\mathrm{g}}{ }^{3} N_{\mathrm{A}}}
$$

where $M, R_{\mathrm{g}}$, and $N_{\mathrm{A}}$ are the molecular weight of polymer, radius of gyration, and Avogadro's number, respectively. $R_{\mathrm{g}}=1.107$ $\times 10^{-2} M^{0.605}$ for PS in toluene. ${ }^{26}$ The overlap concentration from semidilute to concentrated solution, $C^{* *}$, is, however, independent of molecular weight and can be estimated from the equation ${ }^{24}$

$$
C^{* *}=\frac{0.77}{[\eta]^{* *}}
$$

where $[\eta]^{* *}=2.5 N_{\mathrm{A}} V_{\mathrm{e}} / M^{* *} ; V_{\mathrm{e}}=(4 \pi / 3) R_{\mathrm{g}, \theta^{3}}$, where $R_{\mathrm{g}, \theta}$ is the unperturbed root-mean-square end-to-end distance of a polymer chain having a molecular weight of $M^{* *}$. For PS, the entanglement MW, $M^{* *}$ is $\sim 20000,{ }^{27}$ and $R_{\mathrm{g}, \theta}$ is $\sim 3 \mathrm{~nm} .{ }^{24}$ As toluene evaporated from the capillary edge in the sphere-onflat geometry, the concentration of the solution front at the contact line gradually increased with time, undergoing from dilute to semidilute to concentrated solution and eventually forming a glassy polymer ring. From eqs 1 and 2, the $C^{*}$ for PS with different molecular weights can be calculated, yielding $C^{*}=36 \mathrm{mg} / \mathrm{mL}$ for PS-60K, $21 \mathrm{mg} / \mathrm{mL}$ for PS-112K, $6.8 \mathrm{mg} /$ $\mathrm{mL}$ for PS-420K, and $3.6 \mathrm{mg} / \mathrm{mL}$ for PS-876K. The $C^{* *}$ for all PS solutions is $90 \mathrm{mg} / \mathrm{mL}$.

On the basis of the values of $C^{*}$ obtained above, we argue that, for PS-60K, the polymer coils cannot overlap because the solution cannot reach such a high $C^{*}$ (i.e., $36 \mathrm{mg} / \mathrm{mL}$ ) during the course of the solvent evaporation. Accordingly, the viscosity (related to the pinning force) of the solution front was so low that no contact line was pinned to reduce the speed of the displacement of the meniscus at the capillary edge (Figure 1). A steady movement of the solution front was resulted in. A thin layer of polymer solution was, thus, left behind. Eventually, isolated polymer dots (Figure 2) were formed due to the rupture of the liquid thin film driven by unfavorable interfacial interaction between liquid-like PS film and the substrate (i.e., possessing a positive Hamaker constant, $A$, for PS, thereby causing thin film unstable and dewetting).

For PS-112K, the $C^{*}$ is relatively low (i.e., $21 \mathrm{mg} / \mathrm{mL}$ ). The polymer coils may overlap, leading to an increase in the viscosity of the solution front (the intrinsic viscosity, $[\eta]$, is proportional to the square root of MW, i.e., $[\eta]=K \sqrt{M} \alpha^{3}$, where $K$ is Mark-Houwink constant and $\alpha$ is chain expansion factor). Thus, the speed of the displacement of the meniscus at the capillary edge decreased during the solvent evaporation and the pinning of the contact line occurred. As a consequence, more polymers were transported by the capillary flow to the capillary edge, thereby forming a ring. The contact angle of the meniscus decreased due to the evaporative loss of the solvent. When the contact angle was smaller than the critical contact angle, at which the capillary force became larger than the pinning force, the solution front jumped inward to a new position. ${ }^{11}$ Repetitive deposition and recession cycles of the contact line in the sphere- 
(a)

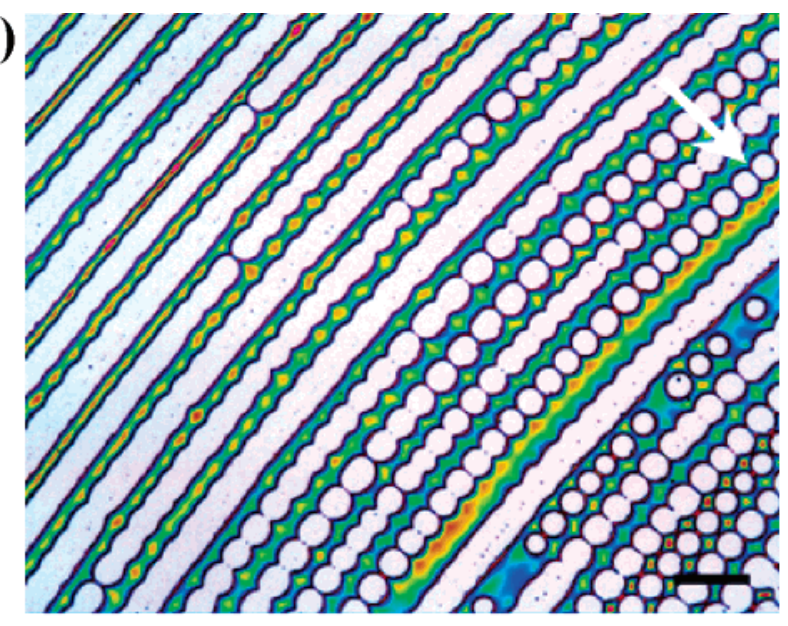

(b)

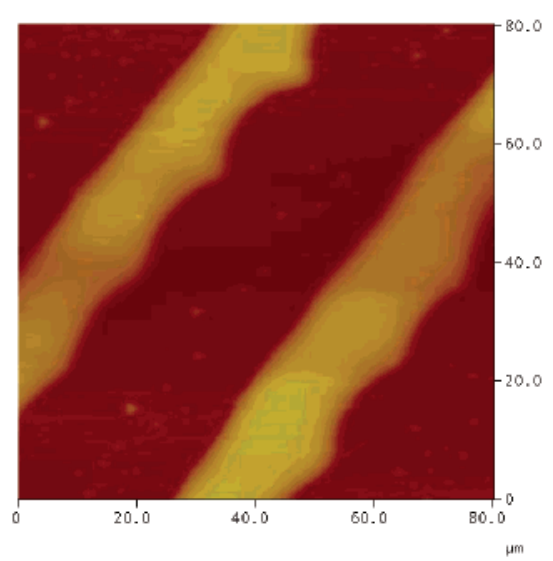

(c)

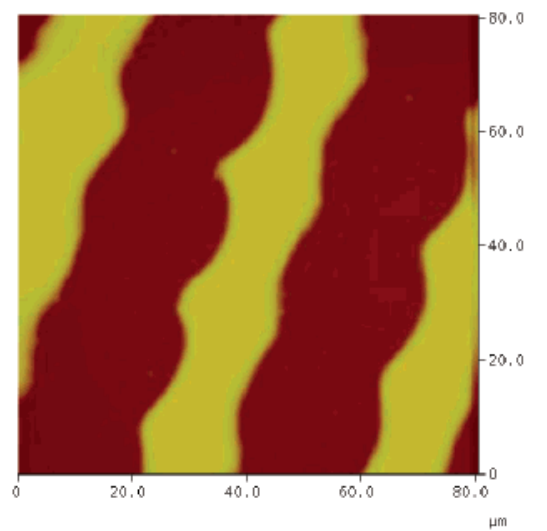

(d)

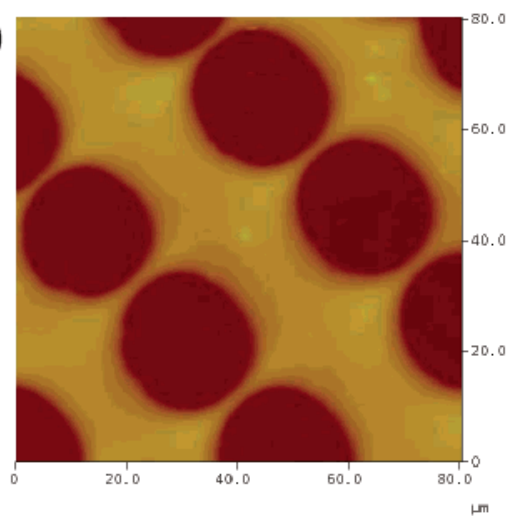

(e)

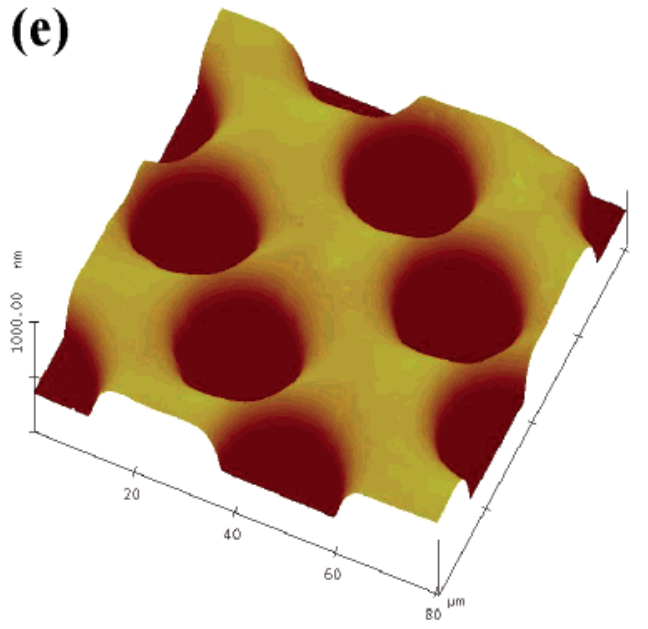

Figure 4. (a) Optical micrograph of surface patterns of PS-420K formed by the drying mediated self-assembly in the sphere-on-flat geometry. The rings, rings with fingers, and punch-hole-like structures (colorful patterns on light gray Si substrate) were obtained. The initial concentration of the PS-420K toluene solution, $c$, is $0.25 \mathrm{mg} / \mathrm{mL}$. Scale bar $=70 \mu \mathrm{m}$. The arrow on the upper right marks the direction of the movement of the solution front. $(b-d) 2 D$ AFM height images of surface patterns, roughly corresponding to the locations in the upper left, middle, and lower right of the optical micrograph in (a). The rings and rings with fingering instabilities are seen in (b) at large $X$. The fingers appeared on both sides of a ring in (c) at intermediate $X$. The punch-hole-like structures were formed when the solution front was closer to the center of sphere/Si contact (i.e., at small $X$ ) as shown in (d). (e) A corresponding 3D AFM height image of (d). The image size is $80 \times 80 \mu \mathrm{m}^{2}$.

on-Si geometry resulted in the formation of concentric rings of PS-112K, as shown in Figure 3. ${ }^{11}$

It is easy to understand that the speed of the solution front decreased more significant when the higher MW PS was used (i.e., PS-420K and PS-876K). The formation of concentric rings is clearly evident (Figure 4). The polymer coils of PS-420K and PS-876K overlapped significantly since $C^{*}$ was only 6.8 and $3.6 \mathrm{mg} / \mathrm{mL}$, respectively. The viscosity increased dramatically during the solvent evaporation as compared to that of PS$112 \mathrm{~K}$. The higher the molecular weight of the polymer, the faster is the rate of increase in viscosity due to the increase in concentration as a result of the solvent evaporation. ${ }^{28}$ This caused a reduction in the speed of solution front, $v,{ }^{29}$ thereby leading to the development of fingering instabilities at a propagating front. ${ }^{23}$ Rings with fingers as well as punch-holelike structures were manifestations of simultaneous occurrence of the "stick-slip" motion of the contact line and the fingering instabilities of rings.

2. Curvature Effect. The mesoscale surface patterns formed by drying the PS toluene solution in the sphere-on-Si geometry can be dynamically tuned by proper choice of the curvature of the sphere. The optical micrograph of the surface pattern produced by drying the $0.25 \mathrm{mg} / \mathrm{mL}$ PS-420K toluene solution is shown in Figure 5a. In this study, the spherical lens with 
(a)

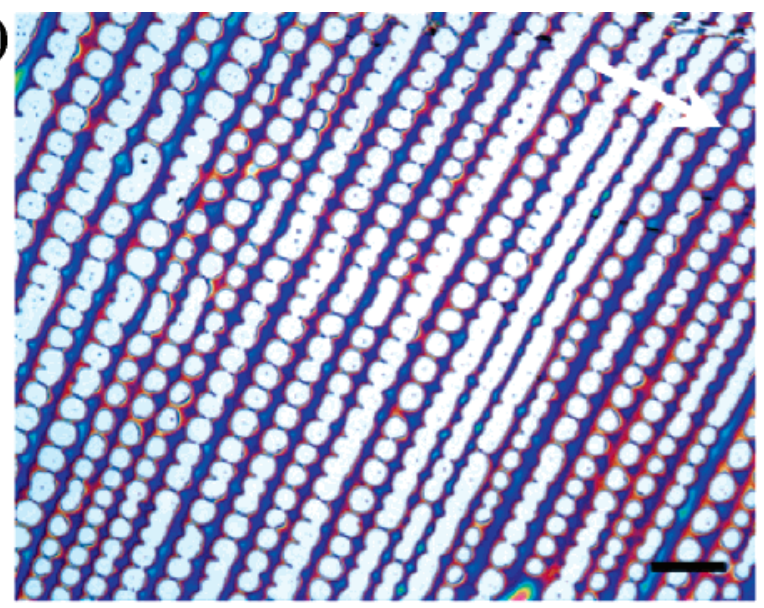

(b)

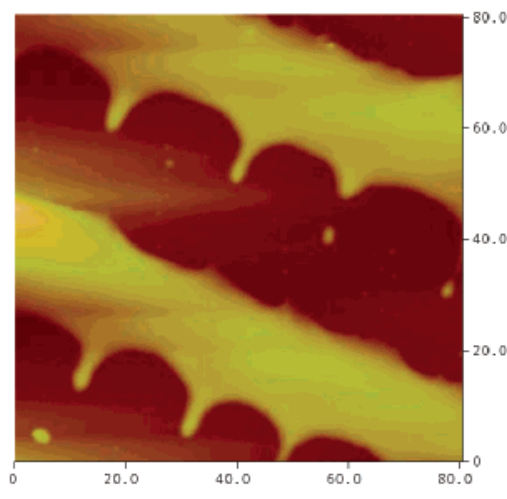

(c)

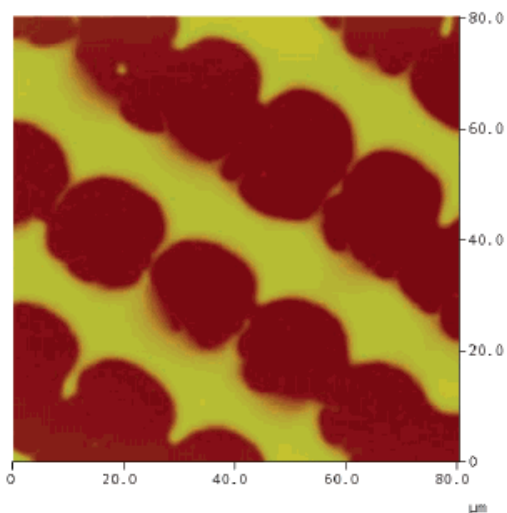

(d)

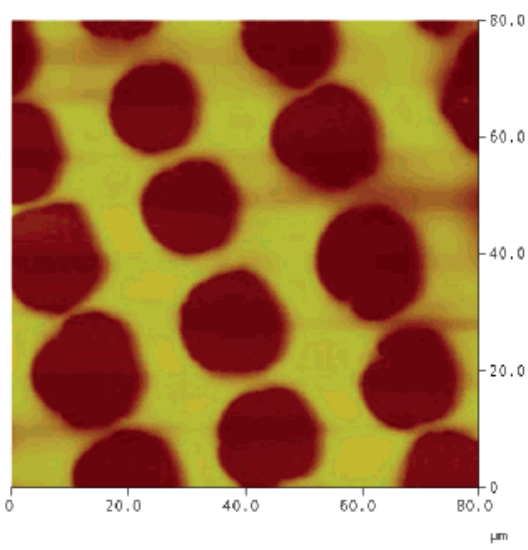

(e)

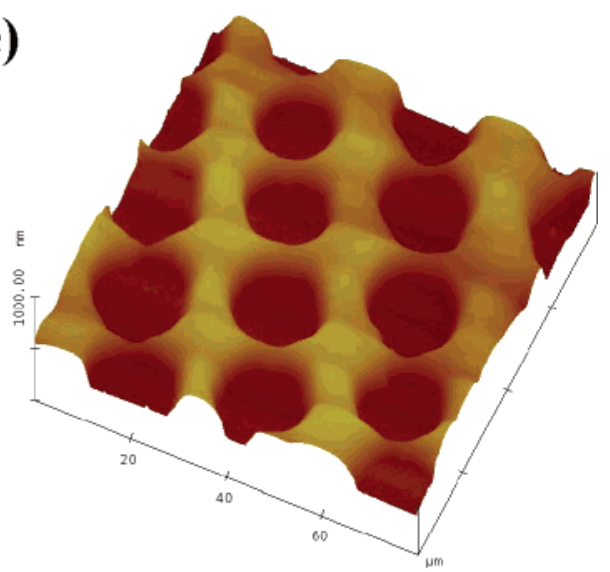

Figure 5. Curvature effect. (a) Optical micrograph of surface patterns of PS-420K produced by the evaporation induced self-assembly of the PS-420K toluene solution confined between the push-pin and Si substrate. The radius of curvature of the push-pin is $2.5 \mathrm{~cm}$. The fingers and the punch-hole-like structures (colorful patterns on light gray Si substrate) are clearly evident. The initial concentration, $c$, is $0.25 \mathrm{mg} / \mathrm{mL}$. Scale bar $=70 \mu \mathrm{m}$. The arrow on the upper right denotes the direction of the motion of the solution front. (b-d) 2D AFM height images of surface patterns, roughly corresponding to the locations in the upper left, middle, and lower right of the optical micrograph in (a). (e) A corresponding 3D AFM height image of (d). The image size is $80 \times 80 \mu \mathrm{m}^{2}$.

curvature of $1 / 2 \mathrm{~cm}^{-1}$ was replaced with a push-pin with curvature of $1 / 2.5 \mathrm{~cm}^{-1}$. Compared to the patterns in Figure 4, an important piece of information was readily gained from Figure 5: more fingers and punch-holes were obtained under the same range of $\Delta X$ (i.e., the same image size in Figures 4 and 5) as the curvature of the sphere decreased. This is simply because the evaporation rate of toluene in the sphere-on-Si geometry slowed down with a large $R$ of the upper sphere. As a result, the displacement of the meniscus at the capillary edge, $v$, reduced. A slower $v$ triggered the earlier onset of fingering instabilities and thus the punch-hole-like structures subsequently (e.g., the punch-hole-like structures formed at $X=3250 \mu \mathrm{m}$ (Figure 5d,e) as compared to those at $X=2950 \mu \mathrm{m}$ (Figure $4 \mathrm{~d}, \mathrm{e})$ ). The uniform zone of the punch-hole-like structure increased. This suggested the possibility of obtaining well- ordered punch-holes structures over larger surface area by manipulating the curvature of the sphere.

The 2D AFM height images, representing the patterns formed at the different stages of the dying process (i.e., progressed from fingering instabilities on the rings to punch-hole-like structures), are shown in Figure $5 \mathrm{~b}-\mathrm{e}$. The characteristic distance between adjacent PS fingers on a ring, $\lambda_{\mathrm{F}}$, and the height of the ring, $h$, were $24.36 \mu \mathrm{m}, 339 \mathrm{~nm}$ at $X=4100 \mu \mathrm{m}$ (Figure 5b), 20.60 $\mu \mathrm{m}, 303 \mathrm{~nm}$ at $X=3450 \mu \mathrm{m}$ (Figure 5c), and $18.99 \mu \mathrm{m}, 289$ $\mathrm{nm}$ at $X=3250 \mu \mathrm{m}$ (Figure $5 \mathrm{~d}, \mathrm{e}$ ). Further scrutiny of the rings having fingers at their edges in Figure $5 b, c$ revealed the formation of isolated dots, residing (Figure 5b,c) or connecting (Figure 5c) between two adjacent fingers, driven by the Rayleigh instability. As the solution front neared the center of the pushpin/Si contact, the mass transportation was facilitated owing to 
a closer distance between two adjacent rings. This led to the formation of more punch-hole-like structures. The average width and height of fingers at the center connecting two adjacent rings are $5.9 \mu \mathrm{m}$ and $228 \mathrm{~nm}$ as measured by AFM (Figure 5d,e).

\section{Conclusion}

Mesoscale polymer patterns were formed by evaporation of a polymer solution in the capillary formed by a sphere resting on a plate (i.e., sphere-on-flat geometry). The change in the polymer molecular weight (MW) led to very pronounced morphological change in the resulting structures. At low MW, the dewetting process occurred, leaving behind randomly distributed dots at the surface. At intermediate MW, the selfassembled concentric rings were formed by repetition of the deposition and recession cycle of the contact lines. At high MW, concentric rings, rings with fingers, and punch-hole-like structures were produced. Furthermore, the change in the radius of curvature of the upper sphere was found to affect the pattern formation. A smaller curvature caused an earlier onset of the formation of fingers and punch-hole-like structures when the high MW PS was utilized as a nonvolatile solute. The present studies provide valuable insights into the rationale of creating intriguing polymer patterns by varying the molecular weight and tuning the radius of curvature of the sphere in the sphereon-flat geometry, which in turn render the control over the solvent evaporation and associated capillary flow.

Acknowledgment. We gratefully acknowledge the support from the $3 \mathrm{M}$ nontenured faculty award and the American Chemical Society Petroleum Research Fund (Grant 42825-G7).

\section{References and Notes}

(1) Nguyen, V. X.; Stebe, K. J. Phys. Rev. Lett. 2002, 88, 164501.

(2) Bormashenko, E.; Pogreb, R.; Musin, A.; Stanevsky, O.; Bormashenko, Y.; Whyman, G.; Barkay, Z. J. Colloid Interface Sci. 2006, 300, 293.
(3) Bormashenko, E.; Pogreb, R.; Musin, A.; Stanevsky, O.; Bormashenko, Y.; Whyman, G.; Gendelman, O.; Barkay, Z. J. Colloid Interface Sci. 2006, 297, 534.

(4) de Gennes, P. G. Eur. Phys. J. E 2001, 6, 421.

(5) Karthaus, O.; Grasjo, L.; Maruyama, N.; Shimomura, M. Chaos 1999, 9, 308.

(6) Hu, H.; Larson, R. G. Langmuir 2005, 21, 3963

(7) Cazabat, A. M.; Heslot, F.; Troian, S. M.; Carles, P. Nature (London) 1990, 346, 824.

(8) Yabu, H.; Shimomura, M. Adv. Funct. Mater. 2005, 15, 575.

(9) Rabani, E.; Reichman, D. R.; Geissler, P. L.; Brus, L. E. Nature (London) 2003, 426, 271.

(10) Lin, Z. Q.; Granick, S. J. Am. Chem. Soc. 2005, 127, 2816.

(11) Xu, J.; Xia, J.; Hong, S. W.; Lin, Z. Q.; Qiu, F.; Yang, Y. L. Phys. Rev. Lett. 2006, 96, 066104.

(12) Hong, S. W.; Xu, J.; Xia, J.; Lin, Z. Q.; Qiu, F.; Yang, Y. L. Chem. Mater. 2005, 17, 6223.

(13) Hong, S. W.; Giri, S.; Lin, V. S. Y.; Lin, Z. Q. Chem. Mater. 2006, $18,5164$.

(14) Hong, S. W.; Xu, J.; Lin, Z. Q. Nano Lett. 2006, 6, 2949

(15) Hong, S. W.; Xia, J.; Lin, Z. Q. Adv. Mater., in press.

(16) Xu, J.; Xia, J.; Lin, Z. Q. Angew. Chem., Int. Ed. 2007, 46, 1860.

(17) Wang, J.; Xia, J.; Hong, S. W.; Qiu, F.; Yang, Y.; Lin, Z. Q. Langmuir, submitted.

(18) Deegan, R. D.; Bakajin, O.; Dupont, T. F.; Huber, G.; Nagel, S. R.; Witten, T. A. Nature (London) 1997, 389, 827.

(19) Deegan, R. D.; Bakajin, O.; Dupont, T. F.; Huber, G.; Nagel, S. R.; Witten, T. A. Phys. Rev. E 2000, 62, 756.

(20) Deegan, R. D. Phys. Rev. E 2000, 61, 475.

(21) Ozawa, K.; Nishitani, E.; Doi, M. Jpn. J. Appl. Phys. 2005, 44, 4229.

(22) Churaev, N. V. Liquid and Vapor flows in Porous Bodies: Surface Phenomena; Gordon and Breach Science: University of Salford, UK, 2000; Vol. 13.

(23) Lyushnin, A. V.; Golovin, A. A.; Pismen, L. M. Phys. Rev. E 2002, 65, 021602.

(24) Ying, Q.; Chu, B. Macromolecules 1987, 20, 362.

(25) Daoud, M.; Cotton, J. P.; Farnoux, B.; Jannink, G.; Sarma, G.; Benoit, H.; Duplessix, R.; Picot, C.; de Gennes, P.-G. Macromolecules 1975, 8, 804.

(26) Stange, T. G.; Mathew, R.; Evans, D. F. Langmuir 1992, 8, 920.

(27) Tsui, O. K. C.; Zhang, H. F. Macromolecules 2001, 34, 9139.

(28) Poh, B. T.; Ong, B. T. Eur. Polym. J. 1984, $20,975$.

(29) Reiter, G.; Sharma, A. Phys. Rev. Lett. 2001, 87, 166103.

MA0700734 